\title{
Efeito das condições de preparação e sinterização sobre a porosidade da hidroxiapatita
}

\section{Effect of preparing and sintering conditions on hydroxyapatite porosity}

\author{
Adalberto Luiz ROSA* \\ Mohammed Youssef SHAREEF** \\ Richard van NOORT***
}

\begin{abstract}
ROSA, A. L.; SHAREEF, M. Y.; van NOORT, R. Efeito das condições de preparação e sinterização sobre a porosidade da hidroxiapatita. Pesqui Odontol Bras, v. 14, n. 3, p. 273-277, jul./set. 2000.

Este estudo foi realizado para verificar o efeito das condições de preparação e sinterização sobre a porosidade de amostras de hidroxiapatita, calculada como porcentagem da densidade teórica da hidroxiapatita. Foram verificados o efeito da carga utilizada para obter as amostras, o tamanho inicial das partículas de hidroxiapatita, a temperatura e o tempo de sinterização. Existiu uma correlação inversa entre carga e porosidade e entre temperatura de sinterização e porosidade, mas nenhuma correlação entre tempo de sinterização e porosidade. Amostras fabricadas com partículas de tamanho maior apresentaram maior porosidade. Foi concluído que para a fabricação de amostras de hidroxiapatita, a combinação entre a carga utilizada para a compactação das partículas e a temperatura de sinterização resulta na produção de amostras com uma ampla variação de porcentagens de porosidade.
\end{abstract}

UNITERMOS: Hidroxiapatitas; Sinterização; Porosidade.

\section{INTRODUÇÃO}

As cerâmicas compostas de cálcio e fosfato, hidroxiapatita e fosfato tricálcio, têm sido utilizadas em várias condições clínicas nas quais é necessário o emprego de enxertos aloplásticos que apresentem biocompatibilidade com o tecido ósseo. Tem sido mostrado que produtos caracterizados como sendo hidroxiapatita pura apresentam biocompatibilidade diferente de produtos formados pela mistura de hidroxiapatita e fosfato tricálcio ${ }^{3}$. Resultados preliminares utilizando cultura de células sugerem que a presença de maior porcentagem de microporos $(<10 \mu \mathrm{m})$ interfere de maneira negativa com a biocompatibilidade da hidroxiapatita $^{4}$. Tais evidências sugerem que o comportamento biológico destas cerâmicas é dependente de vários fatores, dentre eles a composição química e as caracteristicas fisicas finais do produto, justificando a necessidade de se investigar a influência de várias etapas do processo de fabricação da hidroxiapatita sobre o produto final.
Estas cerâmicas podem ser produzidas por uma série complexa e demorada de reações de síntese utilizando, principalmente, carbonato de cálcio e ácido fosfórico. Ao final dessas reações de síntese, obtêm-se as cerâmicas na forma de um pó, isto é, constituídas por um aglomerado de partículas em simples justaposição, mantidas juntas por ligações muito fracas. Nessas condições, as cerâmicas apresentam quase nenhuma propriedade mecânica e essa forma é utilizada para a preparação de amostras, chamadas de "verdes", que posteriormente são submetidas ao processo de sinterização.

As cerâmicas "verdes" podem ser preparadas de diferentes maneiras, em geral, exercendo-se uma carga sobre uma certa quantidade de pó a fim de se obter uma amostra compactada. As técnicas mais comumente utilizadas para isso são: pressão uniaxial, vazamento em moldes (onde não se utiliza carga), pressão uniaxial em altas temperaturas e pressão isostática em altas temperaturas ${ }^{5}$. Nessas

\footnotetext{
* Professor Doutor do Departamento de Cirurgia e Traumatologia Buco-Maxilo-Facial e Periodontia da Faculdade de Odontologia de Ribeirão Preto da USP.

** Pesquisador; *** Professor Titular - Department of Restorative Dentistry, School of Clinical Dentistry, University of Sheffield, Inglaterra.
} 
ROSA, A. L.; SHAREEF, M. Y.; van NOORT, R. Efeito das condições de preparação e sinterização sobre a porosidade da hidroxiapatita.

Pesqui Odontol Bras, v. 14, n. 3, p. 273-277, jul./set. 2000.

duas últimas, a preparação de amostras e sinterização são efetuadas ao mesmo tempo.

A sinterização é o tratamento térmico que é dado às amostras de cerâmica "verde". Nesse processo ocorre a progressiva transição daquele estado de aglomeração, partículas em simples justaposição, para uma unidade na qual as partículas fundem-se umas com as outras. Durante esse processo, ocorrem várias modificações nas cerâmicas, tais como: diminuição da área de superfície, diminuição do volume da amostra, aumento da fase cristalina e aumento das propriedades mecâni$\operatorname{cas}^{1}$. A diminuição do volume da amostra se dá como conseqüência da densificação da cerâmica durante a sinterização, a qual, por sua vez, determina o nivel de porosidade.

Este estudo foi realizado para verificar a influência das condições de preparação e sinterização sobre a porosidade de amostras de hidroxiapatita. Especificamente, foram verificados o efeito da carga utilizada para obter as amostras, o tamanho inicial das partículas de hidroxiapatita, a temperatura e o tempo de sinterização.

\section{MATERIAL E MÉTODOS}

A hidroxiapatita utilizada foi obtida de Plasma Biotal Ltd., Tideswell, UK. Tendo sido mostrado anteriormente que essa hidroxiapatita apresenta alto grau de pureza comparada com outras hidroxiapatitas disponiveis ${ }^{6}$.

Todas as amostras ( $\mathrm{n}=5$ para cada um dos parâmetros avaliados) foram preparadas utilizando a técnica de pressão uniaxial. Para tanto, foi utilizado um molde cilindrico de aço carbono apresentando uma cavidade no centro com $13,5 \mathrm{~mm}$ de diâmetro. Dentro dessa cavidade, por um lado, se encaixava um cilindro sobre o qual foram colocadas as porções de hidroxiapatita, enquanto pelo outro se encaixava outro cilindro sobre o qual foram exercidas as cargas. A fim de obter amostras relativamente uniformes, foi padronizado colocar a mesma quantidade de hidroxiapatita equivalente a, aproximadamente, 0,60 g de pó.

As cargas foram exercidas por meio de uma prensa hidráulica operada manualmente (Graseby Specac) com capacidade para até $15 \mathrm{t}$. Na preparação das amostras, após atingir a carga desejada, a mesma foi mantida por $10 \mathrm{~s}$. A sinterização foi feita utilizando-se um forno Lenton Thermal Designs (UAF 15/5) programado para incrementos de temperatura de $10^{\circ} \mathrm{C} / \mathrm{min}$ até atingir a temperatura desejada e pelo tempo desejado. Após o que, o forno desligava-se automaticamente e as amostras foram deixadas esfriar espontaneamente até a temperatura ambiente.
A fim de verificar o efeito da carga exercida na preparação sobre a porosidade das amostras, foram utilizadas cargas de 1 a 5 t e as amostras foram sinterizadas sob as mesmas condições $\left(1.250^{\circ} \mathrm{C}\right.$ por 3 horas). Para estudar o efeito do tamanho das partículas sobre a porosidade das amostras sinterizadas, foram preparadas amostras de hidroxiapatita nas formas de pó fino e aglomerada, que apresenta partículas maiores. O pó fino foi aquele fornecido pelo fabricante, enquanto a hidroxiapatita aglomerada foi obtida pela mistura de pó fino e outro chamado de granulado, que consistia no pó fino calcinado (mantido a $200^{\circ} \mathrm{C}$ por 1 hora). Para obtenção da hidroxiapatita aglomerada, ambos os tipos de hidroxiapatita foram misturados em água deionizada na proporção, relativa ao peso, de $60 \%$ do granulado para $40 \%$ do fino. Para mantê-los juntos foi adicionado um ligante orgânico (Carbowax). Desse processo resultou a formação de uma pasta, que foi posta para secar em estufa a $100^{\circ} \mathrm{C}$ por 24 horas, após o que, essa mistura foi regranulada utilizando-se gral e pistilo. Os aglomerados formados por esse processo foram classificados utilizando-se peneiras com malhas de $250 \mu \mathrm{m}$ e $500 \mu \mathrm{m}$, obtendo-se, assim, duas amostras de hidroxiapatita aglomerada: uma, com partículas de tamanho $<250 \mu \mathrm{m}$, e outra, com partículas de tamanho $<500 \mu \mathrm{m}$.

A fim de verificar a influência da temperatura e do tempo de sinterização sobre a porosidade das amostras, foram utilizadas diferentes temperaturas e diferentes tempos de sinterização para amostras produzidas empregando-se apenas o pó fino e a mesma carga (2 t).

A porosidade das amostras foi calculada como porcentagem da densidade teórica da hidroxiapatita, que é $3,156 \mathrm{~g} / \mathrm{cm}^{3}$. Para tanto, foram medidos o diâmetro e a altura de todas as amostras, assim como os seus respectivos pesos. Estas medidas foram utilizadas para calcular a densidade das amostras (D), a partir da fórmula abaixo:

$$
\begin{aligned}
& D=4 . P / d^{2} \cdot \pi \cdot h \text {, onde: } \\
& P=\text { peso; } d=\text { diâmetro e } h=\text { altura. }
\end{aligned}
$$

A partir da densidade da amostra, a porcentagem de porosidade foi calculada em relação à densidade teórica da hidroxiapatita $\left(3,156 \mathrm{~g} / \mathrm{cm}^{3}\right)$, utilizando-se a fórmula abaixo:

$$
\% \text { Porosidade }=[1-(D / 3,156)] .100
$$

A análise estatística dos dados foi feita por meio do cálculo do coeficiente de correlação, para verificar a relação entre a carga aplicada e a porcentagem de porosidade, e por meio da análise de variância (ANOVA), seguida pelo teste de Tukey, para 
ROSA, A. L.; SHAREEF, M. Y.; van NOORT, R. Efeito das condições de preparação e sinterização sobre a porosidade da hidroxiapatita.

Pesqui Odontol Bras, v. 14, n. 3, p. 273-277, jul./set. 2000.

verificar a influência do tamanho das partículas sobre a porosidade.

\section{RESULTADOS}

O efeito da pressão utilizada para a produção de amostras, bem como a influência do tamanho das partículas sobre a porosidade, é mostrado no Gráfico 1. A um aumento da carga correspondeu uma diminuição da porcentagem de porosidade, observada para o pó fino $(\mathrm{r}=-0,93 ; \mathrm{t}=-11,98$; $\mathrm{gl}=23 ; \mathrm{p}<0,0001)$, aglomerado $<250 \mu \mathrm{m}$ $(\mathrm{r}=-0,93 ; \mathrm{t}=-12,24 ; \mathrm{gl}=23 ; \mathrm{p}<0,0001)$ e aglomerado $<500 \mu \mathrm{m}(\mathrm{r}=-0,94 ; \mathrm{t}=-13,82 ; \mathrm{gl}=23$; $\mathrm{p}<0,0001)$. A ANOVA mostrou que sobre a porosidade das amostras houve efeitos da carga $(\mathrm{F}=560,28 ; \mathrm{gl}=4 ; \mathrm{p}<0,0001)$, do tamanho da partícula $(\mathrm{F}=274,63 ; \mathrm{gl}=3 ; \mathrm{p}<0,0001)$ e da interação carga versus partícula $(\mathrm{F}=3,1 ; \mathrm{gl}=8$;

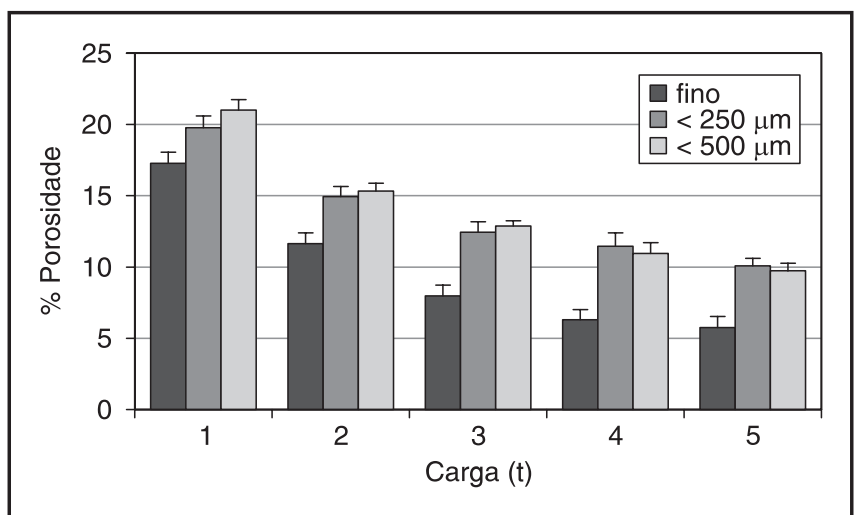

GRÁFICO 1 - Porcentagem de porosidade de amostras de hidroxiapatita preparadas com pós de diferentes tamanhos, utilizando cargas variáveis e sinterizadas nas mesmas condições. Dados mostrados como média \pm desvio-padrão $(\mathrm{n}=5)$.

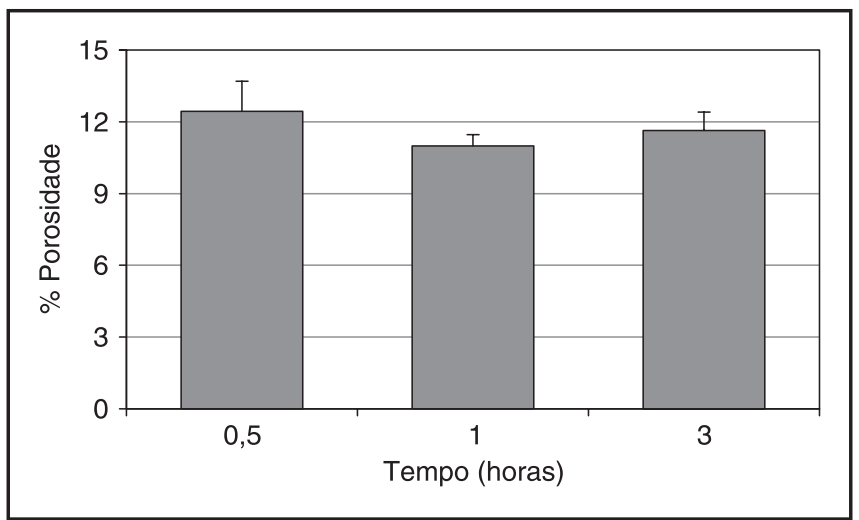

GRÁFICO 2 - Porcentagem de porosidade de amostras de hidroxiapatita sinterizadas por diferentes intervalos de tempo a $1.250^{\circ} \mathrm{C}$. Dados mostrados como média \pm desvio-padrão $(\mathrm{n}=5)$. $\mathrm{p}<0,006)$. A ANOVA, seguida pelo teste de Tukey, comparando-se o efeito do tamanho das partículas para cada uma das cargas utilizadas, mostrou que a utilização do pó fino sempre resultou em amostras com menor porosidade do que aquelas produzidas com a utilização de qualquer um dos aglomerados. Não houve diferença estatisticamente significante entre os dois tipos de aglomerados.

O Gráfico 2 mostra o efeito do tempo de sinterização sobre a porcentagem de porosidade das amostras. Não foi observada correlação entre tempo de sinterização e porosidade das amostras $(\mathrm{r}=-0,32 ; \mathrm{t}=-1,23 ; \mathrm{gl}=13 ; \mathrm{p}=0,24)$.

O Gráfico 3 mostra o efeito da temperatura de sinterização sobre a porcentagem de porosidade das amostras. Pode ser observado que, com o aumento da temperatura ocorreu uma redução na porosidade das amostras $(\mathrm{r}=-0,96 ; \mathrm{t}=-11,78$; $\mathrm{gl}=13 ; \mathrm{p}<0,01)$.

\section{DISCUSSÃO}

Os resultados obtidos mostram o efeito das condições de preparação pelo método da pressão uniaxial e sinterização sobre a porosidade de amostras de hidroxiapatita.

As porcentagens de porosidade encontradas para as amostras deviam estar próximas da porcentagem verdadeira existente nestas amostras. Embora tenha sido utilizado um método envolvendo várias medidas e cálculos, foi demonstrado anteriormente que as porcentagens de porosidade obtidas por este método estão sempre próximas daquelas obtidas por métodos padrões, o que também indica que a grande maioria dos poros é interconectada ${ }^{6,7}$.

Em relação à pressão uniaxial, o aumento da carga utilizada para preparar as amostras resul-

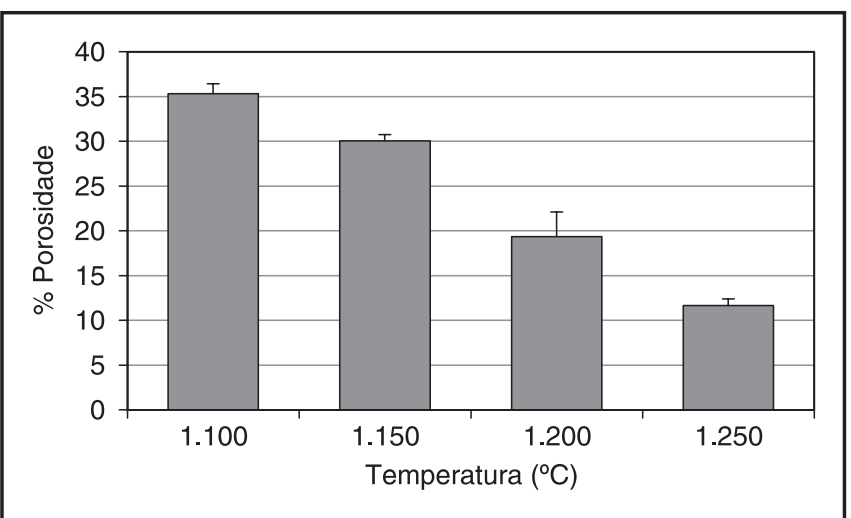

GRÁFICO 3 - Porcentagem de porosidade de amostras de hidroxiapatita sinterizadas utilizando diferentes temperaturas por 3 horas. Dados mostrados como média \pm desvio-padrão $(\mathrm{n}=5)$. 
ROSA, A. L.; SHAREEF, M. Y.; van NOORT, R. Efeito das condições de preparação e sinterização sobre a porosidade da hidroxiapatita.

Pesqui Odontol Bras, v. 14, n. 3, p. 273-277, jul./set. 2000.

tou em uma redução de sua porosidade. Esta relação inversa entre aumento da carga e porcentagem de porosidade foi observada para todas as amostras, independentemente do tamanho das partículas utilizadas para sua preparação, visto que essa relação foi observada tanto para aquelas amostras preparadas com pó fino, como para aquelas preparadas a partir de aglomerados. Entretanto, considerando-se a mesma carga, as amostras preparadas com aglomerados sempre apresentaram porosidade maior do que aquelas preparadas com pó fino, evidenciando-se o efeito do tamanho das partículas sobre a porosidade. Isto se deve ao fato de que na preparação ocorre um empacotamento mais próximo de partículas menores e mais frouxo de partículas maiores; dessa forma, as amostras "verdes" já apresentavam porosidades diferentes ${ }^{5}$. Por essa razão, seria esperado que aglomerados $<500 \mu \mathrm{m}$ apresentassem porosidade maior que daqueles de $<250 \mu \mathrm{m}$. No entanto, isto não aconteceu, porque em ambos os aglomerados havia uma quantidade indeterminada de partículas pequenas, que podem ter produzido um padrão de empacotamento similar, resultando na produção de cerâmicas "verdes" similares em porosidade. Ou ainda, como as ligações que mantêm o tamanho dos aglomerados são fracas, as mesmas podem ter sido facilmente vencidas pelas cargas exercidas para a produção das amostras, também resultando no mesmo tipo de empacotamento, apesar das diferenças nos tamanhos iniciais das partículas. Isto significa que o aumento do tamanho das partículas, ao menos pelo método utilizado nesse estudo, não se constitui em estratégia adequada para produzir amostras mais porosas.

Quanto às condições de sinterização, foi observado que o tempo em que as amostras são mantidas na temperatura de sinterização não interfere no padrão de porosidade. Embora neste estudo tenha sido usada uma variação pequena do tempo de sinterização, isto está de acordo com as obser- vações de outros autores. Enquanto SHAREEF 5 (1991) mostrou tal efeito para amostras de hidroxiapatita, HUBBARD ${ }^{1}$ (1974) mostrou o mesmo efeito na preparação de amostras de fosfato tricálcio.

A temperatura de sinterização apresentou efeito bastante evidente sobre a porosidade das amostras, sendo que, ao aumento da temperatura correspondeu uma redução na porosidade das amostras. Tendo sido mostrado anteriormente que amostras sinterizadas a temperaturas menores do que as utilizadas nesse estudo apresentaram maior porcentagem de porosidade ${ }^{5}$. No entanto, JARCHO et al. ${ }^{2}$ (1976) mostraram que sinterizar hidroxiapatitas a temperaturas menores do que $1.100^{\circ} \mathrm{C}$ resulta em cerâmicas não completamente sinterizadas e, especificamente para a hidroxiapatita utilizada neste estudo, temperaturas maiores do que $1.250^{\circ} \mathrm{C}$ levam a uma degradação com formação de outros produtos à base de cálcio e fosfato, diferentes da hidroxiapatita (dados não publicados). Portanto, devido ao fato de que as alterações na temperatura de sinterização levam a alterações no produto final, tal parâmetro deve ser criteriosamente utilizado com o objetivo de produzir mais ou menos porosidade.

\section{CONCLUSÕES}

Pôde-se concluir que, para a fabricação de amostras de hidroxiapatita, a combinação entre a carga utilizada para a compactação das partículas e a temperatura de sinterização resulta na produção de amostras com uma ampla variação de porcentagens de porosidade. Por outro lado, a utilização de partículas de tamanhos maiores tem um efeito mais limitado sobre a porosidade final das amostras.

\section{AGRADECIMENTOS}

Este trabalho foi patrocinado pela Fundação de Amparo à Pesquisa do Estado de São Paulo (FAPESP), Proc. nº. 95/3190-5.

ROSA, A. L.; SHAREEF, M. Y.; van NOORT, R. Effect of preparing and sintering conditions on hydroxyapatite porosity. Pesqui Odontol Bras, v. 14, n. 3, p. 273-277, jul./set. 2000.

The aim of this study was to evaluate the effect of powder processing and sintering conditions on the resultant porosity of hydroxyapatite, which was calculated as percentage of the theoretical density of hydroxyapatite. The parameters evaluated were the effect of load during uniaxial pressing, the initial size of the particles of hydroxyapatite, and sintering temperature and time. There was an inverse correlation between load and porosity, and between sintering temperature and porosity, but there was no correlation between sintering time and porosity. An increase in the size of the particles resulted in greater porosity. It was concluded that a combination of loading conditions and sintering temperature can provide hydroxyapatite with a wide range of porosities.

UNITERMS: Hydroxyapatites; Sintering; Porosity. 
ROSA, A. L.; SHAREEF, M. Y.; van NOORT, R. Efeito das condições de preparação e sinterização sobre a porosidade da hidroxiapatita.

Pesqui Odontol Bras, v. 14, n. 3, p. 273-277, jul./set. 2000.

\section{REFERÊNCIAS BIBLIOGRÁFICAS}

1. HUBBARD, W. G. Physiological calcium phosphates as orthopedic biomaterials. Milwaukee, 1974. 222 p. Thesis (PhD) - Marquette University.

2. JARCHO, M.; BOLEN, C. H.; THOMAS, M. B.; BOBICK, J.; KAY, J. F.; DOREMUS, R. H. Hydroxylapatite synthesis and characterization in dense polycrystalline form. $\mathbf{J}$ Mater Sci, v. 11, n. 11, p. 2027-2035, 1976.

3. NERY, E. B.; LE GEROS, R. Z.; LYNCH, K. L.; LEE, K. Tissue response to biphasic calcium phosphate ceramic with different ratios of HA/ $\beta$-TCP in periodontal osseous defects. J Periodontol, v. 63, n. 9, p. 729-735, Sep. 1992.
4. ROSA, A. L.; HATTON, P. V.; van NOORT, R. The influence of porosity on the in vitro biocompatibility of hydroxyapatite. J Dent Res, v. 76, n. 5, p. 1081, May 1997.

5. SHAREEF, M. Y. Fabrication, characterization and fracture behaviour of machineable hydroxyapatite ceramics. Sheffield, 1991. 143 p. Thesis (PhD) - University of Sheffield.

6. SHAREEF, M. Y.; MESSER, P. F.; van NOORT, R. Fabrication, characterization and fracture study of a machinable hydroxyapatite ceramic. Biomaterials, v. 14, n. 1 , p. 69-75, Jan. 1993.

7. SHAREEF, M. Y.; MESSER, P. F.; van NOORT, R. Slip cast machineable hydroxyapatite ceramics. Br Ceram Proc, v. 49, p. 121-130, 1992
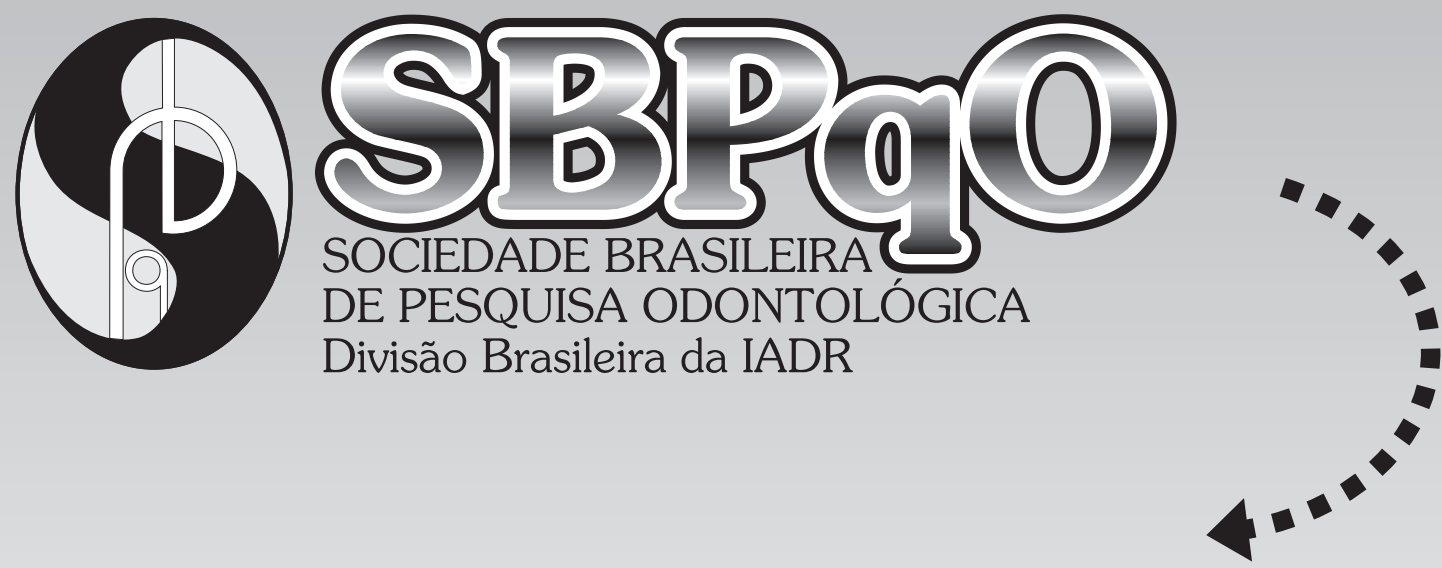

\section{Visite a página da SBPqO na Internet: http://www.sbpqo.org.br}

\section{SBPqO - Av. Professor Lineu Prestes, 2.227 \\ CEP 05508-900 - Cidade Universitária - São Paulo - SP Tel./Fax: 0**11-3818-7855 - E-mail: sbpqo@fo.usp.br}

